\title{
ARQUITETURA DA INFORMAÇÃO: REVISÃO INTEGRATIVA EM BASES DE DADOS DE CIÊNCIA DA INFORMAÇÃO
}

\author{
INFORMATION ARCHITECTURE: INTEGRATIVE \\ REVIEW ON INFORMATION SCIENCE DATABASES
}

\author{
Carin Cunha Rocha ${ }^{a}$ \\ Virgínia Bentes Pinto ${ }^{b}$ \\ Priscila Barros Davidc
}

\begin{abstract}
RESUMO
Introdução: $O$ advento das tecnologias digitais de informação e comunicação propiciou o surgimento de grande quantidade de informação e pouca organização. A arquitetura da informação surgiu nesse cenário com o objetivo de planejar, organizar e projetar os ambientes informacionais de maneira que o usuário encontre a informação que ele procura. Objetivo: Esta pesquisa analisa a presença da arquitetura da informação na literatura científica referente à ciência da informação, pautada na seguinte questão: de que modo a arquitetura da informação está sendo investigada nos artigos científicos da área de Ciência da Informação? Metodologia: Trata-se de uma pesquisa exploratória baseada na revisão integrativa, cuja literatura foi investigada a partir das seguintes fontes de informação: Base de Dados Referencial de Artigos de Periódicos em Ciência da Informação (BRAPCI), Scopus e Library and Information Sciense Abastracts (LISA), contemplando o período de 2000 ao primeiro semestre de 2019. A estratégia de busca pautou-se no termo "arquitetura da informação". A partir dos resultados, foi realizada a leitura dos títulos, resumos, palavras-chave e, em alguns casos, a leitura dos textos completos. Resultados: Setenta e três artigos encontrados sobre a temática da arquitetura da informação foram agrupados nas categorias: arquitetura da informação conectada a diversas áreas, arquitetura da informação e ambientes digitais, arquitetura da informação pervasiva, arquitetura da informação e ciência da informação, usuários, área da saúde, arquitetura da informação e usabilidade, ontologias, metadados. Conclusões: Esse estudo possibilitou a confirmação de que a arquitetura da informação é interdisciplinar, possibilitando conexões com outras áreas do conhecimento.
\end{abstract}

\footnotetext{
a Mestranda do Programa de Pós-Graduação em Ciência da Informação da Universidade Federal do Ceará (UFC). Bibliotecária da Universidade Federal do Maranhão (UFMA). E-mail: carinrocha@hotmail.com

b Doutora em Sciences de l'Information et de la Communication- Institut des Communications et des Média (ICM) da Université Stendhal-Grenoble. Professora da Universidade Federal do Ceará (UFC). E-mail: bentespinto@yahoo.com.br

c Doutora em Educação pela Universidade Federal do Ceará (UFC). Professora do Programa de Pós-Graduação em Ciência da Informação e do Bacharelado em Sistemas e Mídias Digitais da Universidade Federal do Ceará (UFC).E-mail: priscila@virtual.ufc.br
} 
Descritores: Arquitetura da informação. Ciência da Informação. Bases de Dados. Revisão integrativa.

\section{INTRODUÇÃO}

Devido às contribuições da ciência e da tecnologia, a sociedade contemporânea vem evoluindo ao longo do tempo, provocando uma série de mudanças inevitáveis e propiciando avanços significativos em todos os campos dos saberes. Essas transformações atingiram não somente a sociedade, mas, também as áreas do conhecimento gerando mais pesquisa, informação, ciência e tecnologia. Esse avanço permitiu maior dinamização do conhecimento e ampliou a exigência por parte dos usuários que procuram por serviços informatizados.

Cabe frisar também que esse contexto tecnológico permitiu que a sociedade criasse um grande volume de informação, mas sem ordem, cuidado ou organização, gerando dificuldade de recuperação aos usuários. Historicamente, em 1975, o arquiteto Richard Saul Wurman como uma reação a essa problemática cunhou as expressões arquitetura da informação e arquiteto da informação. Para o autor havia muita informação e pouca organização. (WURMAN, 1991). Wurman (1997, p. 76) avança a reflexão sobre a temática e defende a arquitetura da informação como o "estudo da organização da informação com o objetivo de permitir ao usuário encontrar seu caminho de navegação em direção ao conhecimento e a compreensão da informação".

Ressalta-se que a arquitetura da informação tem o objetivo de planejar, organizar e projetar os ambientes digitais permitindo a recuperação da informação e visando à satisfação do usuário. Foi definida por Camargo e Vidotti (2011, p. 24) como

[...] uma área do conhecimento que oferece uma base teórica para tratar aspectos informacionais, estruturais, navegacionais, funcionais e visuais de ambientes informacionais digitais, por meio de um conjunto de procedimentos metodológicos a fim de auxiliar no desenvolvimento e no aumento da usabilidade de tais ambientes e de seus conteúdos.

Na literatura, a arquitetura da informação se configura como complexa e multidisciplinar. Tal complexidade refere-se a essa visão interdisciplinar, 
dialogando com outras áreas e disciplinas. Na visão de Oliveira, Vidotti e Bentes (2015, p. 43).

[...] os valores de universalidade e certeza têm dado lugar à pluralidade e à complexidade. Nessa conjuntura, a arquitetura da informação se estabelece com uma natureza inter/transdisciplinar, com seus métodos, modelos e teorias derivados do diálogo com outras disciplinas.

Nessa perspectiva, alguns estudos já contribuíram para o entendimento da complexidade da arquitetura da informação. Como por exemplo, Macedo (2005, p. 15) que propôs a construção de um conceito amplo para a arquitetura da informação ao "estabelecer sua abrangência temática, identificar seu status científico e sintetizar os principais processos que a definem como uma prática". A autora considerou aspectos epistemológicos, científicos e práticos que contribuem para um entendimento da arquitetura da informação e conseguiu perceber sua relação com outras áreas do conhecimento como a ciência da informação, a ciência da computação, a ergonomia e a usabilidade.

Além desse estudo, Oliveira, Vidotti e Bentes Pinto (2015) se apoiaram em uma pesquisa teórica por meio de um viés epistemológico traçando as origens da arquitetura da informação e realizando uma contextualização espaçotemporal. Os autores a discutem sob quatro aspectos: arquitetural, sistêmica, informacional e pervasiva. "A abordagem arquitetural gera uma arquitetura da informação com fundações interdisciplinares na arquitetura e no design". (OLIVEIRA; VIDOTTI; BENTES PINTO, 2015, p. 52). O paradigma sistêmico age fornecendo modos de pensar que resultam de um fundamento teórico dos sistemas e de uma necessidade de atuação no campo dos sistemas de informação. $O$ aspecto informacional orienta os estudos e as práticas da arquitetura da informação em uma relação direta com o campo da Biblioteconomia e da Ciência da Informação. Por fim, quanto ao paradigma pervasivo, que possui relação com a tecnologia, os autores o defendem como "uma abordagem atual que estuda as ecologias informacionais complexas, sobretudo os processos de hibridização dos lugares humanos, digitais e nãodigitais em que os sujeitos vivem, trabalham e divertem-se". (OLIVEIRA; VIDOTTI; BENTES PINTO, 2015, p. 74).

Através desses estudos, percebe-se que a arquitetura da informação 
engloba experiências e conhecimentos de outras áreas para atingir seus objetivos. Portanto, a realização de uma revisão integrativa nos permite visualizar essa relação, pois muitos artigos na literatura abordam várias temáticas relacionadas à arquitetura da informação, inclusive em conexão com outras áreas do conhecimento.

Uma revisão integrativa é uma "ampla abordagem metodológica referente às revisões, permitindo a inclusão de estudos experimentais e nãoexperimentais para uma compreensão completa do fenômeno analisado". (SOUZA; SILVA; CARVALHO, 2010, p. 103). "O termo integrativa tem origem na integração de opiniões, conceitos ou ideias provenientes das pesquisas utilizadas no método" (BOTELHO; CUNHA; MACEDO, 2011, p. 127). Os autores enfatizam também que uma revisão integrativa pode ser realizada em outras áreas do saber e não somente na área da saúde e educação. Pode-se perceber, portanto, que uma revisão integrativa pode ser usada quando se quer realizar uma síntese e análise do conhecimento científico já produzido sobre o tema investigado.

Assim, tomando como pergunta de partida "De que modo a arquitetura da informação está sendo investigada nos artigos científicos da área de ciência da informação?", definimos como objetivo analisar a presença da arquitetura da informação na literatura científica, referente à ciência da informação.

O artigo está estruturado da seguinte forma: inicialmente, são feitas algumas considerações gerais sobre a arquitetura da informação. Em seguida, detalha-se a metodologia utilizada, demonstrando que o levantamento da literatura foi realizado nas seguintes bases de dados da área de Ciência da Informação: a Base de Dados Referencial de Artigos de Periódicos em Ciência da Informação (BRAPCI), Scopus e Library and Information Sciense Abastracts (LISA), no período de 2000 ao primeiro semestre de 2019. Finalmente, apresentam-se os resultados e as discussões.

\section{CONSIDERAÇÕES SOBRE A ARQUITETURA DA INFORMAÇÃO}

Inicialmente, para entender o que é arquitetura da informação faz-se necessário uma comparação com a vida cotidiana. Tudo na nossa vida tem 
sinalização, tem organização, por exemplo, quando vamos a um supermercado. O supermercado é todo sinalizado, organizado, é dividido em seções e, mesmo sem conhecer aquele ambiente, facilmente encontra-se o produto que se deseja. No ambiente digital também funciona dessa forma: as informações devem ser sinalizadas e organizadas de maneira que o usuário encontre a informação que ele procura, ou seja, que facilite a recuperação da informação.

Apesar de o termo arquitetura da informação ter sido cunhado por Richard Wurmam, na década de 1970, sua consolidação se efetivou com os bibliotecários Louis Rosenfeld e Peter Morville, após a publicação da obra Information architecture for the world wide web, em 1998, permitindo maior visibilidade à mesma. A versão atual desta obra, publicada em 2015, aborda a arquitetura da informação não somente para a web e inclui a participação do autor Jorge Arango. Para esses autores, a Arquitetura da Informação se constitui como

O projeto estrutural de ambientes de informação partilhada.

A combinação de organização, rotulação e esquemas de navegação dentro de sites e intranets.

A arte e a ciência de moldar produtos e experiências de informação para apoiar usabilidade, encontrabilidade e compreensibilidade.

Uma disciplina emergente e uma comunidade prática, focada em trazer para o contexto digital os princípios de design e arquitetura. (ROSENFELD; MORVILLE; ARANGO, 2015, p. 31, tradução nossa)

Na literatura, existem vários conceitos, considerações e abordagens para a arquitetura da informação, mas optou-se por eleger esta proposta, pois constitui uma forma mais abrangente e coerente do ponto de vista teórico e que busca uma melhor compreensão sobre os reais objetivos da arquitetura da informação, além de apresentar seu caráter interdisciplinar. Ela abrange a arquitetura da informação como elemento estrutural para o ambiente informacional digital e que apoia a usabilidade de modo que o usuário possa se deslocar com facilidade nesse ambiente, haja vista que se estrutura por meio da combinação de quatro sistemas (organização, navegação, rotulagem e busca) e que contempla o conteúdo informacional como elemento fundamental para o acesso à informação.

Ainda nesse sentido, consideramos que outras contribuições são importantes, como a de Ziews (2000, p. 11 apud ROBREDO, 2008, p. 123) que 
defende que a arquitetura da informação é a "arte e a ciência de organizar a informação para ajudar as pessoas a satisfazer suas necessidades de informação de forma efetiva (...) o que implica organizar, navegar, marcar e buscar mecanismos nos sistemas de informação".

Em seu glossário disponível online, Hagedorn (2000, p. 5) compreende a arquitetura da informação como "a arte e ciência da organização da informação para a satisfação de necessidades de informação. A arquitetura da informação envolve investigação, análise, projeto e implementação".

Já na visão de Macedo (2005, p. 143) a arquitetura da informação se configura como

[...] uma metodologia de 'desenho' que se aplica a qualquer 'ambiente informacional', sendo este compreendido como um espaço localizado em um 'contexto', constituídos por 'conteúdos' em fluxo; que serve a uma comunidade de 'usuários'. A finalidade da arquitetura da informação é, portanto, viabilizar o fluxo efetivo de informações por meio do desenho de 'ambientes informacionais'.

Resmini e Rosati (2011, p. 19) explicam que "a arquitetura da informação é uma prática profissional e um campo de estudos focados na solução dos problemas básicos de acesso e uso da vasta quantidade de informações disponíveis hoje".

Especificamente, Rosenfeld, Morville e Arango (2015) afirmam que a mesma é formada por elementos ou componentes essenciais que são interdependentes, cada qual composto por regras próprias, ou seja, são elementos básicos que atuam de forma integrada na interação com o usuário. São eles: sistemas de organização, sistemas de navegação, sistemas de rotulação e sistemas de busca.

O sistema de organização define as regras de classificação e ordenação das informações que serão apresentadas, ou seja, organizam a informação de maneira que ajude o usuário a encontrar o que precisa para atingir seu objetivo. O sistema de navegação especifica a forma de se mover através do ambiente informacional. Podem ser de navegação principal ou embutida ou incorporada e suplementar ou remota. O sistema de rotulação define signos verbais e visuais para cada elemento informativo e de suporte à navegação do usuário, são representados pelos rótulos que podem ser textuais ou icônicos. O sistema de 
busca determina as perguntas que os usuários podem fazer e as respostas que irá obter nas bases de dados. Podem utilizar a linguagem natural ou os operadores booleanos. (ROSENFELD; MORVILLE; ARANGO, 2015; AGNER, 2009).

A combinação desses componentes contribui com um ambiente digital planejado e organizado, que facilita as atividades de navegação e pesquisa, conduzindo o usuário a uma boa recuperação da informação, resultando na satisfação do mesmo.

É relevante mencionar os pilares da arquitetura da informação formados por: usuário, conteúdo e contexto, os quais Rosenfeld, Morville e Arango (2015, p. 39, grifo nosso) denominam de ecologia da informação.

Usamos o conceito de 'ecologia da informação' composto por usuários, conteúdo e contexto, para abordar as dependências complexas que existem. E nos baseamos em nosso diagrama fiel de Venn para ajudar as pessoas a visualizar e entender os relacionamentos. Os três círculos ilustram a natureza interdependente dos usuários, conteúdo e contexto dentro de uma complexa e adaptativa ecologia da informação.

Percebe-se que nesta tríade, o usuário é peça fundamental e está presente na base da arquitetura da informação (Figura 1).

Figura 1 - Três pilares da Arquitetura da Informação

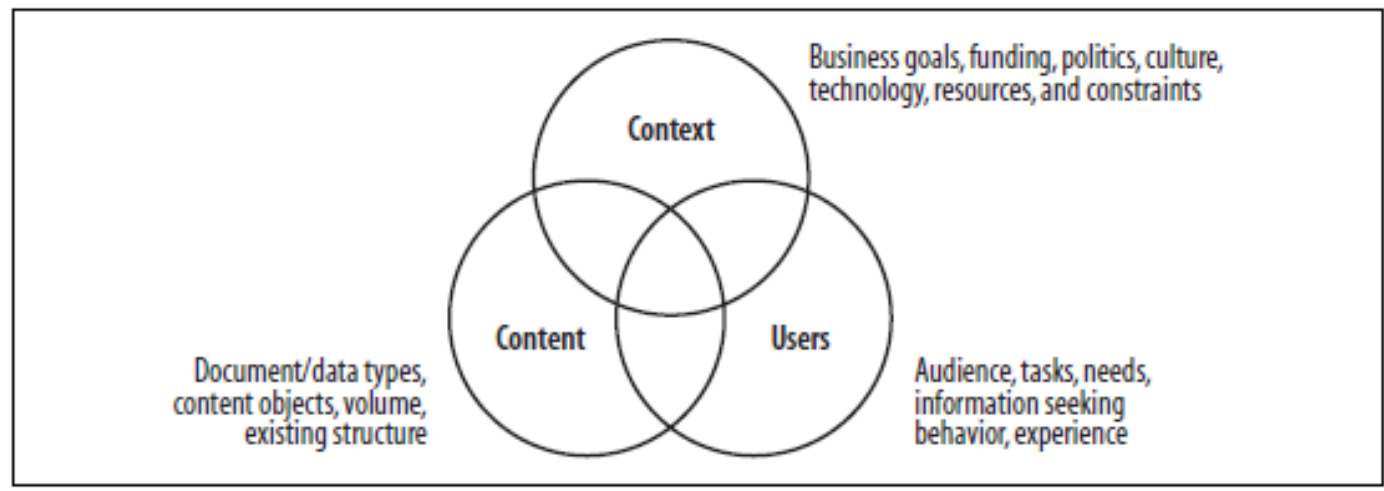

Fonte: Rosenfeld, Morville e Arango (2015, p. 39)

Quer dizer, a arquitetura da informação busca compreender essas três dimensões. Enquanto os usuários são o público-alvo para a informação, incluindo suas necessidades, seus comportamentos e suas experiências; 0 conteúdo são as informações que se pretende disponibilizar. Por sua vez, o contexto compreende os objetivos do site, assim como o ambiente no qual ele se encontra. 


\section{METODOLOGIA}

Conforme mencionado na introdução deste artigo, esta pesquisa está pautada na revisão integrativa da literatura. Consideramos que esse tipo de revisão é uma inovação da revisão de literatura tradicional sendo conceituada por Whittemore e Knafl (2005, p. 546) como uma metodologia especifica "que resume a literatura empírica ou teórica do passado visando a compreensão mais abrangente de um fenômeno ou problema de uma área em particular." As revisões integrativas "apresentam o estado da ciência, contribuem para o desenvolvimento da teoria e têm aplicabilidade direta à prática e à política."

$\mathrm{Na}$ visão de Botelho, Cunha e Macedo (2011), a revisão integrativa é estruturada nas seguintes etapas: identificação do tema e seleção da questão de pesquisa; estabelecimento dos critérios de inclusão e exclusão, identificação dos estudos pré-selecionados e selecionados; categorização dos estudos selecionados; análise e interpretação dos resultados; apresentação da revisão/síntese do conhecimento.

A questão norteadora desta pesquisa é averiguar de que modo a arquitetura da informação está sendo investigada nos artigos científicos da área de ciência da informação. Para tanto nos pautamos na revisão integrativa da literatura cientifica publicada sobre a arquitetura da informação.

O levantamento na literatura foi realizado nas seguintes bases de dados da área de Ciência da Informação: Base de Dados Referencial de Artigos de Periódicos em Ciência da Informação (BRAPCl), Scopus e Library and Information Sciense Abastracts (LISA), no período de 2000 até o primeiro semestre de 2019.

A BRAPCl${ }^{1}$ é um produto do projeto de pesquisa "Opções metodológicas em pesquisa: a contribuição da área da informação para a produção de saberes no ensino superior". Em sua página na internet apresenta como objetivo subsidiar estudos e propostas na área de Ciência da Informação, fundamentando-se em atividades planejadas institucionalmente. Com esse propósito, foram identificados os títulos de periódicos na área de Ciência da

\footnotetext{
${ }^{1}$ http://www.brapci.inf.br/
} 
Informação e indexados seus artigos, constituindo a base de dados referenciais.

A base de dados Scopus ${ }^{2}$ é uma base referencial da editora Elsevier. Indexa títulos acadêmicos revisados por pares, títulos de acesso livre, anais de conferências, publicações comerciais, séries de livros, páginas web de conteúdo científico e patentes de escritórios. Constitui um banco de dados de resumos e citações da literatura oferecendo um panorama abrangente da produção mundial de pesquisas nas áreas de ciência, tecnologia, medicina, ciências sociais, artes e humanidades. Caracteriza-se por disponibilizar ferramentas inteligentes para monitorar, analisar e visualizar pesquisas.

Já a $L I S A^{3}$ constitui uma base de dados internacional criada pela Cambrigde Scientific Abastracts que abrande assuntos de Biblioteconomia, Ciência da Informação e afins. Seu objetivo é proporcionar conteúdo bibliográfico dessas áreas por meio de publicações, recuperação e tecnologia da informação.

O mapeamento dos artigos nessas bases foi pautada na estratégia de busca "arquitetura da informação", com a finalidade de recuperar termos compostos, por isso utilizou-se o termo entre aspas. Em seguida, foi realizada a leitura dos títulos dos artigos, resumos, palavras-chave e, em alguns casos, a leitura dos textos completos. Os dados foram extraídos e organizados em uma planilha contendo: o nome da base de dados, o título do artigo, nome do autor ou autores, título do periódico, ano de publicação do artigo e as palavras-chave referente à arquitetura da informação.

\section{RESULTADOS}

No total, excluindo-se os artigos que se repetiam, foram recuperados 73 documentos que abordaram a temática da arquitetura da informação: 47 artigos na BRAPCI, 18 artigos na base de dados Scopus e oito artigos na base LISA. A grande variedade de assuntos encontrada permitiu-nos reunir os artigos por categorias relacionadas à arquitetura da informação, utilizando a terminologia “Temáticas", conforme Quadro 1.

\footnotetext{
2 https://www-scopus.ez11.periodicos.capes.gov.br/search/form.uri?display=basic

${ }^{3} \mathrm{https}: / /$ search-proquest.ez11.periodicos.capes.gov.br/lisa/index
} 
Quadro 1 - Temáticas sobre arquitetura da informação abordadas em artigos das bases de dados de Ciência da Informação

\begin{tabular}{|l|c|}
\hline \multicolumn{1}{|c|}{ Temáticas } & $\begin{array}{c}\text { Quantidade de } \\
\text { artigos }\end{array}$ \\
\hline Arquitetura da Informação conectada a áreas diversas & 22 \\
\hline Análise de Ambientes Digitais & 15 \\
\hline Arquitetura da Informação Pervasiva & 10 \\
\hline Arquitetura da Informação e Ciência da Informação & 10 \\
\hline Usuários & 5 \\
\hline Área da Saúde & 5 \\
\hline Arquitetura da Informação e Usabilidade & 3 \\
\hline Ontologias & 2 \\
\hline Metadados & 1 \\
\hline
\end{tabular}

Fonte: Dados da Pesquisa

Os artigos que abordaram a arquitetura da informação em conexões com outras áreas do conhecimento (direito, administração, marketing, economia, lógica, semiótica, psicologia e outras áreas) foram nomeados nesta pesquisa com temática Arquitetura da informação conectada a áreas diversas; já os artigos que fizeram uma análise de ambiente digitais de acordo com os elementos da arquitetura da informação propostos por Rosenfeld, Morville e Arango (2015) (sistema de organização, de navegação, de rotulação e de busca), foram dispostos na temática Análise de Ambientes Digitais; os estudos que contemplaram a arquitetura da informação pervasiva foram reunidos na temática Arquitetura da Informação Pervasiva; os artigos que relacionaram a Arquitetura da Informação à Ciência da Informação foram organizados na temática Arquitetura da Informação e Ciência da Informação; os artigos que analisaram a arquitetura da informação com foco no usuário foram organizados na temática Usuários; os artigos que contemplaram a arquitetura da informação na área da saúde estão listados na temática Área da Saúde; os artigos que relacionaram a Arquitetura da Informação à Usabilidade foram reunidos na temática Arquitetura da Informação e Usabilidade; os artigos que explicaram a arquitetura da informação por meio de Ontologias foram reunidos na temática de mesmo nome, e por fim, apenas um artigo foi encontrado com a temática Arquitetura da Informação e Metadados. 


\section{DISCUSSÃO}

A disposição dos resultados da pesquisa em grupos de temáticas permitiu realizar uma análise crítica dos artigos coletados.

\subsection{Arquitetura da Informação Conectada A Diversas Áreas}

Algumas produções científicas encontradas na pesquisa evidenciam a arquitetura da informação como uma área multidisciplinar possuindo conexões com outras áreas do conhecimento, de acordo com alguns artigos dispostos no Quadro 2.

\section{Quadro 2 - Títulos de artigos que abordaram a arquitetura da informação em conexões com outras áreas do conhecimento}

Tecnologias da web semântica na arquitetura da informação

A arquitetura da informação em plataformas colaborativas como suporte para a gestão da inteligência coletiva nas organizações

Os Sistemas de Recomendação, Arquitetura da Informação e a Encontrabilidade da Informação

Da necessidade de princípios de arquitetura da informação para a internet das coisas Arquitetura da informação no ambiente digital: avaliando as relações com o Direito da Propriedade Intelectual

A gestão dos espaços informacionais em ambientes de arquitetura da informação organizacional

Arquitetura da informação e mensuração do desempenho: Um estudo na indústria de artefatos e utensílios de plásticos no estado de São Paulo

Web, Web Semântica e Web $\quad$ Pragmática: um posicionamento da Arquitetura da Informação

O Direito da Propriedade Intelectual: relações com os entregáveis da Arquitetura da Informação

Arquitetura da informação para uma economia da informação

A arquitetura da informação à luz da teoria de Piaget: uma possibilidade epistemológica para a gestão do conhecimento

Arquitetura da informação e enterprise architecture: questões terminológicas

A Arquivística e a Arquitetura da Informação: uma análise interdisciplinar

O exercício da arquitetura da informação na Web: reflexões relativas à Lei dos Direitos Autorais

O marketing e a arquitetura da informação para web no contexto do processo de mediação da informação

O posicionamento da arquitetura da informação na governança de TI

Uma proposta de posicionamento da arquitetura da informação no gerenciamento de serviços de $\mathrm{TI}$

Aspectos da Arquitetura da Informação envolvidos no mapeamento de processos em Organizações Militares sob a perspectiva semiótica

Interfaces entre a arquitetura da informação e a semiótica 
O diálogo entre arquitetura da informação e linguística textual como facilitadores ao acesso e uso das tecnologias

Arquitetura da Informação Multimodal

Análise de uma Arquitetura da Informação associada a Multimodalidade na

capacitação de profissionais de alto desempenho

\section{Fonte: Dados da Pesquisa}

A respeito dessas conexões, a arquitetura da informação envolve a análise, o design e a implementação de espaços informacionais como sites, bancos de dados, bibliotecas. Conforme Agner (2009), sua visibilidade coincidiu com o momento crítico da internet a partir da segunda metade dos anos 90. Ainda na opinião desse autor "a arquitetura da informação pode ser vista como a união de três campos tradicionais: a tecnologia, 0 design e 0 jornalismo/redação." (AGNER, 2009, p. 79)

Portanto, a arquitetura da informação se conecta com outras áreas do conhecimento. Em função dessas conexões, ela deve ser entendida com um termo "guarda-chuva" na concepção de Dillon e Turnbull (2005), sob o qual coexistem várias outras autodenominações de profissionais e de pesquisadores. (Figura 2).

Figura 2 - Modelo conceitual da Arquitetura da Informação

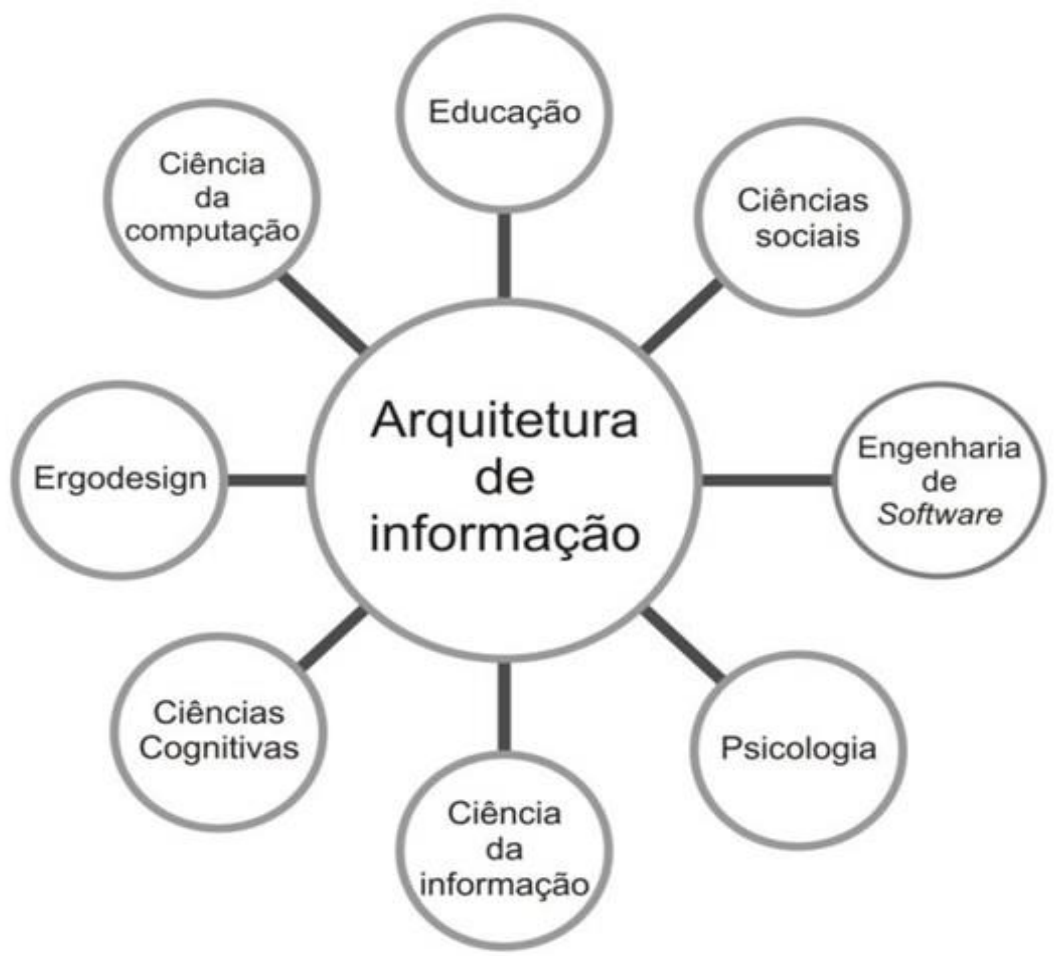

Fonte: Agner (2009, p. 77). 
Compartilham desse pensamento Resmini e Rosati (2011) ao afirmarem que a arquitetura da informação tem suas raízes em um grande número de diferentes disciplinas: em formação de design, design visual, biblioteca e ciência da informação, psicologia cognitiva, arquitetura e provavelmente alguns outros.

\title{
5.2 AnÁlise da Arquitetura da Informação em Ambientes Digitais
}

Foi possível perceber que muitos artigos vinculam a arquitetura da informação aos sistemas propostos pelos autores Rosenfeld, Morville e Arango (2015): sistema de organização, de navegação, de rotulação e de busca, realizando essa análise em ambientes digitais. Os títulos de artigos encontrados estão apresentados no Quadro 3.

\section{Quadro 3 - Títulos de artigos que abordaram a arquitetura da informação em análise de ambientes digitais}

\begin{abstract}
Arquitetura da informação em repositórios institucionais: desafios e perspectivas Organização da informação no cenário radiofônico de universidades paranaenses a partir das recomendações da arquitetura da informação

Biblioteca digital de teses e dissertações do IBICT: uma análise sob a ótica da arquitetura da informação

Arquitetura da informação no contexto de gênero: uma análise do website da Secretaria Especial de Políticas para Mulheres

Arquitetura da informação no website Geledés: a mulher negra em foco

Elementos de arquitetura da informação no Repositório Eletrônico Institucional da UFPB
\end{abstract}

A dimensão tecnológica da gestão do conhecimento e a contribuição da arquitetura da informação: uma análise da plataforma podio

Arquitetura da informação e sintaxe das linguagens imagéticas no website Guia Gay Floripa

Reflexões sobre Arquitetura da Informação para dispositivos móveis

Análise sobre sistemas de busca na perspectiva da arquitetura da informação em ambiente de cordéis

Arquitetura da Informação em sites de Pró-Reitorias de Graduação: um enfoque nas Instituições Estaduais de Ensino Superior do Paraná

Portais de sistemas de gestão participativa de governo: possibilidades de estruturação sob a perspectiva da Arquitetura da Informação

Acesso à informação financeira em websites: uma análise do portal da transparência da Prefeitura Municipal de Marcação/PB sob a perspectiva da arquitetura da informação

Navegabilidade em portais: estudo com usuários dos portais dos jornais O Norte e Jornal da Paraíba

Arquitetura da informação com enfoque semiótico no guia gay São Paulo

Fonte: Dados da Pesquisa 


\subsection{Arquitetura da Informação Pervasiva}

Com a ampliação do escopo da arquitetura da informação para arquitetura da informação pervasiva, algumas produções científicas ilustram essa nova abordagem, conforme os títulos de artigos encontrados expostos no Quadro 4.

\section{Quadro 4 - Títulos de artigos que abordaram a arquitetura da informação pervasiva}

\begin{tabular}{|l}
\hline Ecossistemas de informação: novo paradigma para a arquitetura da informação \\
\hline $\begin{array}{l}\text { Gestão da informação em ambientes híbridos: condições de apoio da arquitetura da } \\
\text { informação }\end{array}$ \\
$\begin{array}{l}\text { Desafios da arquitetura da informação pervasiva: Reduzindo a desorientação, } \\
\text { aumentando a legibilidade e wayfinding }\end{array}$ \\
$\begin{array}{l}\text { Gestão da Informação em ambientes } \\
\text { apoio da Arquitetura da Informação }\end{array}$ \\
\hline $\begin{array}{l}\text { Navegando na Arquitetura da Informação Pervasiva: o artigo científico como Ecologia } \\
\text { Informacional Complexa }\end{array}$ \\
\hline $\begin{array}{l}\text { Proposta para produção de livros eletrônicos com foco na arquitetura da informação } \\
\text { pervasiva }\end{array}$ \\
\hline $\begin{array}{l}\text { Contribuições metodológicas para a avaliação da arquitetura da informação pervasiva } \\
\text { em repositórios digitais institucionais }\end{array}$ \\
\hline $\begin{array}{l}\text { Arquitetura da informação pervasiva: uma evolução das interfaces computacionais } \\
\text { responsivas }\end{array}$ \\
\hline $\begin{array}{l}\text { Arquitetura da informação pervasiva e encontrabilidade da informação: instrumento para } \\
\text { a avaliação de ambientes informacionais híbridos }\end{array}$ \\
\hline Arquitetura da Informação pervasiva: desvendando as heurísticas de Resmini e Rosati \\
\hline
\end{tabular}

Fonte: Dados da Pesquisa

A arquitetura da informação pervasiva "é uma abordagem atual que estuda as ecologias informacionais complexas, sobretudo os processos de hibridização dos lugares humanos, digitais e não-digitais em que os sujeitos vivem, trabalham e divertem-se". (OLIVEIRA; VIDOTTI; BENTES PINTO, 2015, p. 74). Ou seja, relaciona a arquitetura da informação diretamente com a tecnologia.

\subsection{ARQUITETURA dA INFORMAÇÃo E CIÊNCIA dA INFORMAÇÃo}

É de suma importância destacar a relação entre a Arquitetura da Informação e a Ciência da Informação, afinal são áreas interdisciplinares que permitem elaborar relevantes produções científicas, como as apresentadas no Quadro 5. 


\section{Quadro 5 - Títulos de artigos que abordaram a relação entre a arquitetura da informação e a ciência da informação}

\begin{tabular}{|l|}
\hline $\begin{array}{l}\text { Estratégias para disseminação do conhecimento organizacional: o papel da } \\
\text { arquitetura da informação }\end{array}$ \\
\hline Arquitetura da informação e o paradigma sócio-cognitivo \\
\hline $\begin{array}{l}\text { Arquitetura da informação em bibliotecas digitais: uma abordagem da Ciência da } \\
\text { Informação e da Biblioteconomia }\end{array}$ \\
\hline $\begin{array}{l}\text { Análise da arquitetura da informação na produção de visualização de dados em } \\
\text { ambiência digital }\end{array}$ \\
\hline Indicadores da produção colaborativa na arquitetura da informação \\
\hline Ciência da Informação e Arquitetura da Informação como referenciais para as Teias \\
Hipertextuais Complexas \\
\hline MAIA - Método de arquitetura da informação aplicada \\
\hline Arquitetura da Informação e Ciência da Informação. Notas de (re)leitura à luz do \\
paradigma pós-custodial, informacional e científico \\
\hline Estruturas de Representação da Informação e seu apoio à Arquitetura da Informação \\
na web: um olhar sobre vocabulários controlados, tesauros e metadados \\
\hline O paradigma social e o tempo do conhecimento interativo: Perspectivas e desafios \\
para a arquitetura da informação pervasiva
\end{tabular}

Fonte: Dados da Pesquisa

A existência de uma relação entre Arquitetura da Informação e Ciência da Informação pode ser constatada na visão de Pádua, Dias e Sousa (2019) que afirmam que a Arquitetura da Informação tem sido objeto de estudos, principalmente na Ciência da Informação porque está oferece instrumentos que simplifiquem e melhorem a estrutura informacional e visual de ambientes informacionais digitais e físicos.

Portanto, há um diálogo entre as duas áreas, particularmente no que diz respeito às temáticas de rotulagem, contexto e usuário, as quais a Ciência da Informação vem contemplando em seus estudos e traz embutida em seu conceito seminal.

\subsection{ARQUITETURA DA INFORMAÇÃO E USUÁRIOS}

Quando se trata de arquitetura da informação, o usuário é peça importante, de acordo com Rosenfeld, Morville e Arango (2015) que abordam o usuário como um dos pilares da arquitetura da informação, assim como o conteúdo e o contexto, conforme já mencionado anteriormente. Nessa perspectiva, foram encontrados na pesquisa artigos que centraram a arquitetura da informação no usuário, conforme exemplificado no Quadro 6. 


\section{Quadro 6 - Títulos de artigos que abordaram a arquitetura da informação centrada no usuário}

Arquitetura da informação em portais de notícias: implicações relacionadas à sobrecarga cognitiva e à desorientação do usuário

Arquitetura da informação em livros: uma aproximação a partir do comportamento de busca dos usuários

Arquitetura da informação no comércio eletrônico de livros no Brasil: dimensões que norteiam a e-satisfação do usuário

Arquitetura da Informação para quê e para quem?: uma reflexão a partir da prática em ambientes informacionais digitais

O papel da arquitetura da informação na produção de sentidos pela abordagem sense-making

Fonte: Dados da Pesquisa

Em conformidade com a visão de Rosenfeld, Morville e Arango (2015); Davenport (1998) defende uma abordagem ecológica para a arquitetura da informação, ao afirmar que assim como acontece com muitas abordagens orientadas para o computador, a arquitetura não chegará a lugar nenhum se não levar em consideração o comportamento e a motivação humanos. Os arquitetos precisam se comunicar com aqueles cujo comportamento será modificado continuamente, devem identificar e ouvir as pessoas que influenciarão a mudança, incluindo os usuários-alvo.

Além do mais, os sites devem ter seus produtos com base na linguagem e no modelo mental do usuário, afinal

[...] ergodesign e a arquitetura de informação trabalham com os processos mentais humanos - os chamados processos cognitivos. Esses processos dizem respeito a como as pessoas apreendem a informação e como constroem conhecimentos. Objetiva-se a mudança conceitual do projeto centrado no sistema para o projeto centrado no usuário. Considero aí sistema a palavra em termos bem amplos: pode estar relacionado também às organizações ou aos sistemas de poder e de controle vigentes. (AGNER, 2009, p. 11)

Tomando como alicerce a arquitetura da informação, a Experiência do Usuário (UX), através da norma ABNT ISO 9241-210 criada em 2011, aborda a ergonomia, a interação humano-sistema e o design centrado em pessoas e sistemas interativos. São as respostas e percepções de uma pessoa resultante do uso de um produto, sistema ou serviço. Essa norma possui seis princípioschave que são: o projeto é baseado no entendimento explícito de usuários, tarefas e ambientes; os usuários estão envolvidos em todo projeto e 
desenvolvimento; o projeto é conduzido e refinado por avaliações centradas no usuário; o processo é iterativo; o projeto aborda toda a experiência do usuário; a equipe de design inclui competências multidisciplinares e perspectivas.

Garret (2011) propõe que os cinco elementos da experiência do usuário, do mais abstrato ao mais concreto, são: estratégia, escopo, estrutura, esqueleto e superfície. É chamado de iceberg da experiência do usuário. A arquitetura da informação entra nesse contexto como design estrutural do espaço da informação para facilitar o acesso intuitivo ao conteúdo. Ou seja, a arquitetura da informação compreende uma parte da experiência do usuário.

Outra contribuição quando se fala em usuário é a da Engenharia Cognitiva. Para Barbosa e Silva (2010, p. 53) "a engenharia cognitiva foi concebida por Donald Norman em 1986 como uma tentativa de aplicar conhecimentos de ciência cognitiva, psicologia cognitiva e fatores humanos ao design e construção de sistemas computacionais". Ou seja, está centrada na relação entre usuário e sistema.

\subsection{Arquitetura da INFormação na Área da SAÚde}

Foi formado um grupo específico que relaciona a arquitetura da informação com a área da saúde devido à quantidade de produções científicas encontradas com importantes contribuições dessas áreas, conforme exemplificado no Quadro 7.

Quadro 7 - Títulos de artigos que abordaram a arquitetura da informação na área da saúde

Definição da arquitetura de informação e de serviços do Registro Eletrônico de Saúde (RES) do Brasil

Pensando a arquitetura da informação pervasiva aplicada ao registro eletrônico de saúde

A arquitetura da informação centrada no usuário: estudo do website da Biblioteca Virtual em Saúde (BVS)

Arquitetura da informação: uma ferramenta de mediação e acesso aos Prontuários eletrônicos do Paciente

Arquitetura da Informação: estudo e análise da base de dados Public Medical (PubMed)

Fonte: Dados da Pesquisa

São pesquisas que envolvem a análise de ambientes digitais de acordo com os sistemas de Rosenfeld, Morville e Arango (2015), arquitetura da informação pervasiva e como ferramenta de mediação voltada para à área da 
saúde.

\subsection{ARQUITETURA DA INFORMAÇÃO E USABILIDADE}

Os trabalhos científicos encontrados e apresentados no Quadro 8 confirmam que a arquitetura da informação é multidisciplinar quando compartilha conteúdos e conhecimentos de várias áreas, como a Interação HumanoComputador (IHC) e quando analisa problemas de usabilidade. (CAMARGO, 2010).

\section{Quadro 8 - Títulos de artigos que abordaram a relação entre a arquitetura da informação e a usabilidade}

O acesso a informações e a contribuição da Arquitetura da informação, usabilidade e acessibilidade

Da informação à compreensão: reflexões sobre arquitetura da informação, usabilidade e acessibilidade no campo da ciência da informação

Arquitetura da informação e principios de usabilidade: proposta de um modelo de análise e desenvolvimento para websites de turismo LGBTQ

Fonte: Dados da Pesquisa

Fazendo uma relação entre as áreas, Camargo (2010) afirma que a usabilidade é um requisito essencial para a elaboração dos elementos essenciais da arquitetura da informação, visando à facilidade de uso pelo usuário, principalmente nas questões ligadas à organização do sistema.

A usabilidade é um dos critérios de qualidade de uso na área de IHC (Interação Humano-Computador) que enfatiza certas características da interação e da interface que as tornam adequadas aos efeitos esperados do uso do sistema. Para tanto, Barbosa e Silva (2010, p. 28) afirmam a usabilidade é um dos critérios de qualidade do uso assim como a experiência do usuário, a acessibilidade e a comunicabilidade.

Portanto, a usabilidade está relacionada à facilidade de aprendizado e uso da interface, bem como à satisfação do usuário em decorrência desse uso (BARBOSA; SILVA, 2010). Tradicionalmente, a usabilidade enfoca a maneira como a utilização de um sistema interativo no ambiente de trabalho é afetado por características do usuário (sua cognição, sua capacidade de agir sobre a interface e sua capacidade de perceber as respostas do sistema). Com a disseminação dos sistemas computacionais interativos em ambientes diferentes 
do trabalho, a usabilidade passou a englobar também as emoções e os sentimentos dos usuários.

A usabilidade compreende um conjunto de fatores que qualificam quão bem uma pessoa pode interagir com um sistema interativo (NIELSEN, 1993). Além disso, "a usabilidade é um atributo de qualidade relacionado à facilidade do uso de algo." (NIELSEN; LORANGER, 2007) O autor considera os seguintes fatores de usabilidade: facilidade de aprendizado, facilidade de recordação, eficiência, segurança no uso e satisfação do usuário.

Com relação à avaliação da usabilidade, existem diversos métodos e técnicas na literatura. Segundo Barbosa e Silva (2010) existem os seguintes métodos de avaliação em IHC: métodos de observações de uso e métodos de inspeção. Os métodos de inspeção permitem ao avaliador inspecionar uma solução de IHC para tentar antever as possíveis consequências de certas decisões de design sobre as experiências de uso. Ou seja, tentam identificar problemas que os usuários podem vir a ter quando interagirem com o sistema, ou seja, problemas potenciais.

\subsection{ARQuitetura da INFORMAÇÃo E ONTOLOGIAS}

Visando a organização e representação do conhecimento, as ontologias também são abordadas quando se fala em arquitetura da informação, conforme os artigos encontrados e especificados no Quadro 9.

\section{Quadro 9 - Títulos de artigos que abordaram a arquitetura da informação através de ontologias}

Ontologia como um artefato da arquitetura da informação para a representação do conhecimento organizacional

InfoArch: uma ontologia para modelar o domínio da Arquitetura da Informação para Web

Fonte: Dados da Pesquisa

A ontologia, originada na Filosofia, é aplicada em várias áreas do conhecimento, entre elas na Ciência da Informação. Uma ontologia é uma especificação explicita de uma conceituação, ou seja, a definição explícita de conceitos e suas relações, propriedades e restrições expressas formalmente. (GRUBER, 1993). Permite especificar uma visão abstrata e simplificada de um 
assunto que se deseja representar.

\subsection{ARQUITETURA dA INFORMAÇÃo E METADAdOS}

Nesta pesquisa, apenas um artigo abordou metadados, conforme disposto no Quadro 10.

\section{Quadro 10 - Título do artigo sobre arquitetura da informação e metadados}

A arquitetura e a recuperação da informação para a melhoria do índice $H$ na Plataforma Lattes

Fonte: Dados da Pesquisa

De acordo com Rosenfeld, Morville e Arango (2015), os sistemas de organização, de navegação, de rotulação e de busca se apoiam nos sistemas de representação da informação como os metadados, os vocabulários controlados e os tesauros. Os metadados são constituídos como termos utilizados para descrever e representar o conteúdo dos objetos como documentos, pessoas, processos e organizações. "Metadados e vocabulários controlados apresentam uma lente fascinante através da qual podemos ver a rede de relacionamentos entre sistemas". (ROSENFELD; MORVILLE; ARANGO, 2015, p. 258). Ou seja, é uma abordagem relevante da arquitetura da informação que compartilha conhecimentos da Biblioteconomia e da Ciência da Informação, fornecendo um aspecto informacional.

Os metadados configuram como importantes elementos na arquitetura da informação, constituídos como termos utilizados para descrever e representar o conteúdo dos objetos como documentos, pessoas, processos e organizações. Conceitualmente, Camargo e Vidotti (2011, p. 161) afirmam que os metadados são "formatos que possibilitam a descrição de documentos digitais, definindo-os como 'dados sobre dados', a fim de descrever e localizar documentos eletrônicos na web de forma mais adequada".

Ampliando esse conceito, na opinião de Souza, Catarino e Santos (1997, p. 94) "metadados são descrições de dados armazenados em banco de dados, ou como é comumente definido 'dados sobre dados a partir de um dicionário digital de dados"”.

Isto é, os metadados são estruturas para organizar a informação. Além 
disso, podem focar tanto o ponto de vista do sistema, facilitando a interoperabilidade, quanto o ponto de vista do usuário, facilitando o mesmo a obter detalhes sobre a informação. Então, os dados foram criados para constituir a informação.

Além disso, os metadados funcionam como mediadores entre o usuário e o documento. Para ilustrar, é pertinente a informação de Ramos, Alcântara e Moresi (2019) ao afirmarem que os metadados são as lentes pelas quais enxergamos a informação, pois conduzem a organização para a obtenção da informação qualificada, conceituada e contextualizada, localizável e com sentido para o negócio, ampliando, em muito, a importância que pode ser derivada a partir da utilização de metadados.

Camargo e Vidotti (2011) apresentam os metadados como um dos recursos essenciais de ambientes informacionais digitais, assim como a acessibilidade, a usabilidade, a política, a interoperabilidade, a preservação, a ferramenta de busca, a personalização e a customização. Para estas autoras, os metadados trazem diversas vantagens aos usuários, pois por meio de uma representação padronizada dos recursos informacionais disponíveis em meio eletrônico, proporcionam o acesso mais amplo aos conteúdos, facilitam a busca, integram e compartilham recursos heterogêneos.

\section{CONCLUSÃO}

Com este estudo, percebeu-se que uma revisão integrativa é mais do que uma síntese da literatura científica, ela permite uma análise profunda das produções científicas coletadas proporcionando amplas discussões. Portanto, o objetivo da pesquisa foi alcançado, mapeando a produção científica sobre arquitetura da informação permitindo uma discussão crítica por meio de diálogos com autores sobre as temáticas encontradas.

Além disso, evidenciou-se que a revisão integrativa foi relevante nesta pesquisa porque possibilitou identificar importantes produções científicas que abordam a arquitetura da informação sob diferentes temáticas nos últimos dezenove anos, o que pode trazer grandes contribuições para outras pesquisas, para a comunidade científica e para a ciência de forma geral. 
Como foi demonstrado, o resultado dessa pesquisa possibilitou a confirmação de que a arquitetura da informação é complexa e multidisciplinar, possuindo conexões com outras áreas do conhecimento.

\section{REFERÊNCIAS}

AGNER, Luiz. Ergodesign e arquitetura da informação: trabalhando com o usuário. 2. ed. Rio de Janeiro: Quartet, 2009.

BARBOSA, Simone Diniz Junqueira; SILVA, Bruno Santana da. Interação humano-computador. Rio de Janeiro: Elsevier, 2010.

BOTELHO, Louise Lira Roedel; CUNHA, Cristiano Castro de Almeida; MACEDO, Marcelo. O método da revisão integrativa nos estudos organizacionais. Gestão e Sociedade, Belo Horizonte, v. 5, n. 11, p. 121-136, maio/ago. 2011. Disponível em:

http://www.gestaoesociedade.org/gestaoesociedade/article/view/1220/906. Acesso em: 15 maio 2019.

CAMARGO, Liriane Soares de Araújo de. Metodologias de desenvolvimento de ambientes digitais a partir dos princípios da arquitetura da informação. 2010. 287f. Tese (Doutorado em Ciência da Informação) - Departamento de Faculdade de Filosofia e Ciências, Universidade Estadual Paulista, Marília, 2010.

CAMARGO, Liriane Soares de Araújo de; VIDOTTI, Silvana Aparecida B. G. Arquitetura da informação: uma abordagem prática para o tratamento de conteúdo e interface em ambientes informacionais digitais. Rio de Janeiro: LTC, 2011.

DAVENPORT, Thomas H. Ecologia da informação. São Paulo: Futura, 1998.

DILLON, Andrew; TURNBULL, Don. Information Architecture. Encyclopedia of Library and Information Science, 2005. Disponível em: https://www.researchgate.net/publication/279439252_Information_Architecture Acesso em: 11 abr. 2019.

GARRET, Jesse Jamnes. The elements of user experience: user-centerd design for the web and beyond. 2. ed. Berkeley, 2011.

GRUBER, T. R. What is an Ontology?, 1993. Disponível em: https://tomgruber.org/writing/ontolingua-kaj-1993.htm Acesso em: 19 maio 2019. 
HAGEDORN, Kat. Information Architecture Glossary. USA, 2000. Disponível em: https://argus-acia.com/white_papers/ia_glossary.pdf. Acesso em 08 jul. 2019.

MACEDO, Flávia Lacerda Oliveira de. Arquitetura da informação: aspectos epistemológicos, científicos e práticos. 2005. 190f. Dissertação (Mestrado em Ciência da Informação) - Departamento de Ciência da Informação e Documentação, Universidade de Brasília, Brasília, 2005.

NIELSEN, Jakob. Usability Engineering. New York, NY: Academic Press, 1993.

NIELSEN, Jakob; LORANGER; Hoa. Usabilidade na web: projetando websites com qualidade. Rio de Janeiro: Elsevier, 2007.

OLIVEIRA, Henry P. C. de; VIDOTTI, Silvana A. B. G.; BENTES PINTO, Virgínia. Arquitetura da informação pervasiva. São Paulo: Editora UNESP; São Paulo: Cultura Acadêmica, 2015, 117p.

PÁDUA, Mariana Cantisani; DIAS, Guilherme Ataíde; SOUSA, Marckson Roberto Ferreira de. Conectando os paradigmas da ciência da informação com a arquitetura da informação pervasiva e a experiência do usuário. Disponível em:

https://www.academia.edu/17804089/conectando_os_paradigmas_da_ci\%c3\% 8ancia_da_informa\%c3\%87\%c3\%83o_com_a_arquitetura_da_informa\%c3\%87 \%c3\%83o_pervasiva_e_a_experi\%c3\%8ancia_do_usu\%c3\%81rio. Acesso em 7 maio 2019 .

RAMOS, Rosana Guedes Cordeiro; ALCÂNTARA, Alexandre; MORESI, Eduardo Amadeu Dutra. Construindo uma arquitetura da informação baseada em metadados. Disponível em: https://www.researchgate.net/publication/228821395_Construindo_uma_Arquit etura_da_Informacao_Baseada_em_Metadados. Acesso em: 8 maio 2019.

RESMINI, Andrea; ROSATI, Lucas. Pervasive information architecture: designing cross-chanel user expeiences. Amsterdã: Elsevier, 2011.

ROBREDO, Jaime. Sobre arquitetura da informação. Revista Ibero-americana de Ciência da Informação, v. 1 n. 2, p. 115-137, jul./dez. 2008.

ROSENFELD, Louis; MORVILLE, Peter; ARANGO, Jorge. Information architecture for the world wide web and beyond. 4 ed. Sebastopol: O'Reilly Media, 2015.

SOUZA, Terezinha Batista de; CATARINO, Maria Elisabete; SANTOS, Paulo César dos. Metadados: catalogando dados na internet. Transinformação, v. 9 , n. 2. p. 93-105, maio/ago. 1997. 
SOUZA, Marcela Tavares de; SILVA, Michelly Dias da; CARVALHO, Rachel de. Revisão integrativa: o que é e como fazer. Einstein, v. 8, 2010.

WHITTEMORE R.; KNAFL K. The integrative review: updated methodology. Journal of Advanced Nursing. v. 52, n. 5, p. 546- 553, 2005. Disponível em: http://citeseerx.ist.psu.edu/viewdoc/download?doi=10.1.1.465.9393\&rep=rep1\&t ype=pdf. Acesso em: 10 jul. 2008.

WURMAN, Richard Saul. Ansiedade de Informação: como transformar informação em compreensão. São Paulo: Cultura Editora Associados, 1991. $235 p$.

\title{
INFORMATION ARCHITECTURE: INTEGRATIVE REVIEW ON INFORMATION SCIENCE DATABASES
}

\begin{abstract}
Introduction: The advent of digital information and communication technologies has led to the emergence of large amounts of information and little organization. Information architecture emerged in this scenario with the objective of planning, organizing and designing informational environments so that the user finds the information he seeks. Objective: This research seeks to analyze the presence of information architecture in the scientific literature related to information science, based on the following question: how is information architecture being investigated in scientific articles in the area of Information Science? Methodology: It is an exploratory research based on the integrative review, and the literature search was performed on the following sources of information: Reference Database of Information Science Journals (BRAPCI), Scopus and Library and Information Sciense Abastracts (LISA), The period covered was 2000 to the first half of 2019. The search strategy was based on the term "information architecture". From the results, the titles, abstracts, keywords were read and, in some cases, the full texts were read. Results: Seventy-three articles found on the subject of information architecture were grouped into the following categories: information architecture connected to various areas, information architecture and digital environments, pervasive information architecture, information architecture and information science, users, health area, information architecture and usability, ontologies, metadata. Conclusions: This study allowed the confirmation that the information architecture is interdisciplinary, allowing connections with other areas of knowledge.
\end{abstract}

Descriptors: Information Architecture. Information Science. Data base. Integrative review.

\section{ARQUITECTURA DE INFORMACIÓN: REVISIÓN INTEGRATIVA DE BASES DE DATOS DE CIENCIAS DE LA INFORMACIÓN}




\section{RESUMEN}

Introducción: el advenimiento de las tecnologías digitales de información y comunicación ha llevado a la aparición de grandes cantidades de información y poca organización. La arquitectura de la información surgió en este escenario con el objetivo de planificar, organizar y diseñar entornos informativos para que el usuario encuentre la información que busca. Objetivo: Esta investigación busca analizar la presencia de la arquitectura de la información en la literatura científica relacionada con la ciencia de la información, basándose en la siguiente pregunta: ¿cómo se investiga la arquitectura de la información en artículos científicos en el área de la ciencia de la información? Metodología: es una investigación exploratoria basada en la revisión integradora, y la búsqueda bibliográfica se realizó en las siguientes fuentes de información: Base de datos de referencia de revistas de ciencias de la información (BRAPCI), Scopus y Library and Information Sciense Abastracts (LISA), El período cubierto fue de 2000 a la primera mitad de 2019. La estrategia de búsqueda se basó en el término "arquitectura de la información". A partir de los resultados, se leyeron los títulos, resúmenes, palabras clave y, en algunos casos, se leyeron los textos completos. Resultados: Setenta y tres artículos encontrados sobre el tema de la arquitectura de la información se agruparon en las siguientes categorías: arquitectura de la información conectada a varias áreas, arquitectura de la información y entornos digitales, arquitectura de la información generalizada, arquitectura de la información y ciencias de la información, usuarios, área de salud, arquitectura de la información y usabilidad, ontologías, metadatos. Conclusiones: Este estudio permitió confirmar que la arquitectura de la información es interdisciplinaria, permitiendo conexiones con otras áreas del conocimiento.

Descriptores: Arquitectura de la información. Ciencia de la información. Bases de datos. Revisión integradora.

Recebido em: 05.10 .2019

Acesso em: 09.06.2020 